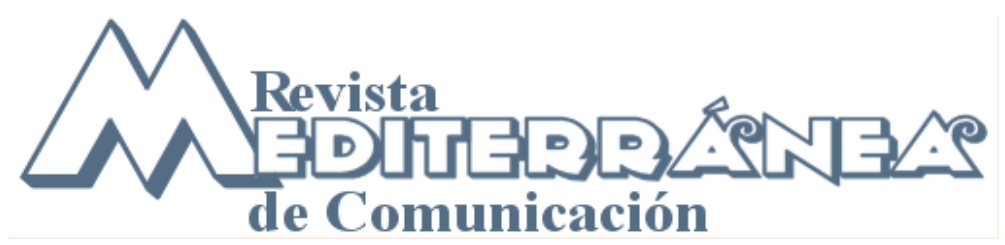

Año 2 (2011), pp. 171-190 ISSN 1989-872X

Fecha de recepción: 15/06/2011; Fecha de publicación: 20/07/2011

\title{
El complejo mundo de la interactividad: emociones y redes sociales
}

\section{The complex world of interactivity: emotions and social networks}

\author{
Dra. Estrella Martínez Rodrigo \\ Facultad de Comunicación y Documentación \\ Universidad de Granada (UGR) \\ emrodrigo@ugr.es \\ Rosario Segura García \\ Facultad de Comunicación y Documentación \\ Universidad de Granada (UGR) \\ rosega@correo.ugr.es \\ Lourdes Sánchez Martín \\ Facultad de Comunicación y Documentación \\ Universidad de Granada (UGR) \\ lousanm@correo.ugr.es
}

\begin{abstract}
Resumen
El objetivo de la presente investigación es discernir cómo debe plantearse un aprendizaje en nuevas tecnologías, en especial, en redes sociales. Para esto, se analizará la dinámica neurológica y psicológica de las emociones y cómo se desenvuelven en el ámbito de las redes sociales. La metodología que emplearemos será acudir a diversos estudios neurológicos y psicológicos actuales, en especial de Antonio Damasio, para analizar posteriormente qué sucede con las emociones cuando nos comunicamos a través de redes sociales, tomando como referente un experimento neurológico de dicho autor en el cerebro de varios voluntarios que muestra la aparición de diversos tipos de emociones. Por último, extrapolaremos este experimento a la aparición de emociones si se utiliza como medio de comunicación las redes sociales y realizaremos algunas conclusiones acerca de la dirección a la que debe tender el aprendizaje de nuevas tecnologías, en especial, redes sociales, teniendo en cuenta dichas observaciones.
\end{abstract}

\section{Abstract}

Forma de citar este artículo: Martínez Rodrigo, E., Segura García, R., Sánchez Martín, (2011) "El complejo mundo de la interactividad: emociones y redes sociales", en Revista Mediterránea de comunicación, 2, pp. 171-190. Recuperado el día de mes de año (añadir enlace) 
The objective of this research is to discern how it should consider learning on new technologies, especially in social networks. For this, we examine the neurological and psychological dynamics of emotions and how they develop in the field of social networks. Our methodology will go to various neurological and psychological studies today, including Antonio Damasio and how to analyze what happens when we communicate emotions through social networks, by reference to a neurological experiment that copyright in the brain several volunteers that shows the appearance of various types of emotions. Finally, we extrapolate this experiment to the appearance of emotions when social networks are used as media and will make some conclusions about the direction you should aim to learn new technologies, especially social networks, taking into account such observations.

\section{Palabras claves}

Redes sociales; interactividad; comunicación; educación; valor moral; psicología.

\section{Key Words}

Social network; interactivity communication; education; moral value; psychology.

Sumario: 1. Introducción. Importancia de las emociones en la actualidad de las ciencias sociales. 2. Objetivo. 3. Metodología. 4. ¿Qué queremos decir cuando pronunciamos la palabra comunicación? 5. Descripción científica de las emociones: elementos neurológicos y psicológicos. 5.1. Anatomía de la emoción. 5.2. Fisiología y psicología de la emoción. Consciencia de emoción. 5.3. Sentimiento y emoción. Sentimiento del sentimiento: consciencia del sentimiento. 6. Interactividad, comunicación y emociones. 6.1. La esencia de la comunicación digital. La interactividad. 6.2. Dinámica de las emociones en redes sociales. 7. Conclusiones. 8. Bibliografía. 9. Notas.

Summary: 1. Introduction. Importance of emotions in social sciences today. 2. Objectives. 3. Methodology. 4. What do we mean when we say the word communication? 5 . Scientific description of emotions: neurological and psychological elements. 5.1. Emotion anatomy. 5.2. Physiology and psychology of emotion. 5.3. Emotion and sentiment. Sentiment of sentiment: Conscience of sentiment. 6. Interactivity, communication and emotions. 6.1. The essence of digital communication. 6.2. Dynamic of emotions in social networks. 7. Conclusions. 8. Bibliography. 9. Notes.

\section{Introducción. Importancia de las emociones en la actualidad de las ciencias sociales.}

El término emoción no ha sido utilizado de modo frecuente hasta el s.XIX. Parece que fue Thomas Brown (1778-1820) quien introdujo el uso más habitual de esta palabra (Dixon, 2003: 109-127), aunque Hume ya realiza un uso temprano del término. Hasta ese momento, los términos más utilizados eran pasión, sentimientos, o afecciones. Emoción, con todas sus connotaciones, es muy utilizada en la psicología del s.XIX especialmente por William James, y por Darwin. También Ernst Mach (1818-1916) que es deudor del empirismo, como él mismo reconoce, aunque no habla explícitamente de emociones, tiene un planteamiento muy similar al de Hume en el tratamiento de las sensaciones, que suponen una base para entender el interés por dicho término en la actualidad (Mach, 1987: 42). 
En el siglo XX, ha habido un interés creciente por el papel de las emociones en la sociedad, pues según algunos sociólogos, había sido olvidado en los tratados tradicionales acerca de la sociedad ${ }^{1}$. Asimismo comienzan a realizarse planteamientos en los cuales las emociones cobran una gran importancia en la explicación de diversos comportamientos sociales, o incluso, dan razones de la evolución de las sociedades capitalistas (Illouz, 2009).

También desde la filosofía, especialmente en el ámbito anglosajón, se ha investigado y revisado el concepto de emoción desde diversos puntos de vista (Nussbaum, 1997), (Goldie, 2000), (Kenny, 1969). Pero la aparición del término emoción no es fortuita, ni azarosa, sino que es el resultado de todo un proceso, en el cual Adam Smith tuvo un papel importante.

Todo ello contribuye a configurar una sensibilidad propia de nuestro tiempo, ya que la sociedad occidental está constituida en gran parte sobre su planteamiento económico -y dicho planteamiento no es ajeno a su filosofía moral- y a su Teoría de los sentimientos morales. La consideración de la noción de pasión de Adam Smith es ya muy diferente a la que usaba la tradición aristotélico-tomista. Esa evolución, mediatizada por el pensamiento cartesiano, que da origen al racionalismo y al empirismo, aporta las claves de por qué en el siglo XIX, la noción de emoción es más usada que la de pasión. Cuando Thomas Brown comienza a usar el término pasión, tiene detrás una tradición empirista, y recoge aportaciones de Hume y Reid, e indirectamente de Smith, ya que éste participa en gran medida del planteamiento de Hume.

A su vez, el gran desarrollo de la neurología en el siglo XX, con importantes figuras como Antonio Damasio, facilita comprender, a nivel fisiológico, cómo se desenvuelven las emociones en el cerebro y explican mejor las conductas relacionadas con ellas.

Con los elementos neurológicos y psicológicos, daremos respuesta a lo que se entiende por emoción y su reflejo en la conducta, con el fin de comprender mejor cómo debe ser el aprendizaje de las tecnologías para que su uso responda a una comunicación humana y no quede en algo desnaturalizado, reducido a mera técnica

\section{Objetivo}

El objetivo de la presente investigación es discernir cómo debe constituirse un aprendizaje mediante las tecnologías, en especial las redes sociales, teniendo en cuenta que las emociones son unas realidades imprescindibles en la comunicación humana, para lo que se analizará la dinámica neurológica y psicológica de las emociones y cómo se desenvolverían en el ámbito interactivo.

\section{Metodología}

La metodología que emplearemos será, en primer lugar, la de acudir a diversos estudios neurológicos actuales, principalmente del doctor Antonio Damasio; en segundo lugar, nos acercaremos a las investigaciones psicológicas más relevantes. Por último, analizaremos cómo se desenvolverían las emociones en redes sociales, apoyándonos en dichos estudios. Con estas observaciones elaboraremos las conclusiones.

\section{4. ¿Qué queremos decir cuando pronunciamos la palabra comunicación?}

El término comunicación se emplea desde numerosos ámbitos del saber, científico o humanístico, y en la vida corriente. Para acotar las diversas definiciones que pueden encontrarse, acudimos a 
Wolton (2005: 22), el cual señala tres sentidos relevantes para una investigación como la que tenemos entre manos.

La primera acepción es entender la comunicación como una experiencia antropológica fundamental en la que hay un intercambio con el otro. La segunda es interpretar el término, a la luz del desarrollo tecnológico del siglo XX y XXI, como un conjunto de técnicas que hacen eficaz la transmisión de información. Por último, puede entenderse la comunicación como una necesidad social funcional, es decir, un intercambio entre sociedades, no sólo entre individuos.

A juicio de Wolton, hay una realidad común que atraviesa estos tres niveles y es la interacción, es decir, tanto en una comunicación directa, como tecnológica, o entre sociedades, es necesario interactuar.

Interactuar no sólo es poner en contacto, sino que supone un intercambio de algo. Si dos individuos van caminando al lado por la calle, uno junto a otro, no interactúan, pero sí en el momento en el que uno pregunta a otro, la hora, por ejemplo.

El tema que tratamos aquí es la comunicación en redes virtuales, para la que la interactividad es un elemento esencial. Normalmente usamos el término interactividad para referirnos a la peculiar relación que se establece entre un emisor y un receptor cuando se utilizan medios tecnológicos o lo que podríamos llamar un sistema: informático, de vídeo u otro.

El grado de interactividad del producto viene definido por la existencia de recursos que permiten que el usuario establezca un proceso de actuación participativa-comunicativa con los materiales. Varios autores ya han destacado desde hace años, lo imprescindible que resulta que las informaciones que se canalizan a través de los recursos multimedia sufran un análisis exhaustivo ante los posibles errores de interpretación por los destinatarios (Estebanel, 2002: 15-25). También se observa la necesidad de una preparación de los usuarios que les capacite para recibir, interpretar y valorar este tipo de mensajes; de este modo, se evitaría que el contenido percibido sea incompleto o erróneo. Las emociones juegan un papel esencial en la adecuada compresión de un mensaje.

La interactividad que nos ocupa es una interactividad propia de una comunicación humana. Aunque se utilicen medios tecnológicos, tanto quien emite como quien recibe el mensaje es un individuo y, por tanto, se mostrarán rasgos típicos de la comunicación interpersonal. Y es aquí es donde entran en juego las emociones. Para poder comprender bien su papel en las tecnologías, se hace necesario, en primer lugar, comprender bien su funcionamiento a nivel neurológico.

\section{Descripción científica de las emociones: elementos neurológicos y psicológicos}

\subsection{Anatomía de la emoción}

Hay algunos aspectos anatómicos a tener en cuenta al analizar la neurología de las emociones en el ser humano. Respecto a los hemisferios, el derecho es dominante para aspectos relacionados con la dimensión espacial de la información recibida, localizados en la corteza parietal dorsal posterior, así como en el giro cingulado. La dominancia del hemisferio izquierdo es para aspectos relacionados con el lenguaje, estando situada esta función en las áreas de Broca y de Wernicke. Por otro lado, existe un módulo relacionado con la conexión memoria-emoción, que estaría situado en las regiones del hipocampo y en el complejo de la amígdala. Asimismo se localiza un módulo funcional de ejecución conductual, en la corteza lateral prefrontal, la corteza orbitofrontal, 
y la corteza parietal posterior. Y por último, se puede determinar un módulo de identificación de caras y objetos, en la corteza lateral temporal.

En estos puntos de referencia, las estructuras cerebrales que mayor atención están recibiendo actualmente en el ámbito del análisis de la emoción, han sido la amígdala y los hemisferios cerebrales.

\subsubsection{Amígdala}

Debido a su localización, y a las conexiones con otras estructuras cerebrales, supone una zona de especial importancia emocional. La amígdala recibe información sensorial de todo tipo, y está en contacto con el hipocampo, el prosencéfalo basal y los ganglios basales, que son estructuras relacionadas con la memoria y la atención, así como con el hipotálamo, que es fundamental para el control de la homeostasis y la regulación neuroendocrina. La amígdala se encuentra implicada tanto en el procesamiento de la estimulación emocional expresiva como en una función de procesamiento emocional más amplio y general, con connotaciones sociales.

\subsubsection{Hemisferios cerebrales}

En cuanto a los hemisferios cerebrales, tradicionalmente el hemisferio izquierdo se considera implicado en aquellos aspectos emocionales que se transmiten a través del lenguaje, o que suponen la descripción verbal de una emoción, mientras que el hemisferio derecho estaría más relacionado con los aspectos emocionales que se transmiten mediante características expresivas y gestuales.

Más recientemente, se ha sugerido la especial relevancia del hemisferio derecho para el procesamiento de la información con connotaciones emocionales, tanto en el caso de seres humanos como en el caso de animales. Diversas investigaciones sugieren que la corteza frontal inferior derecha está implicada en el procesamiento de las señales emocionales, tanto visuales como acústicas, pudiendo defender la existencia de una asimetría hemisférica, al menos en la zona frontal inferior, en relación con el procesamiento de la información emocional; y ponen de relieve que el hemisferio derecho juega un papel más importante en la percepción e interpretación de la entonación emocional.

La implicación del hemisferio derecho en el procesamiento emocional es clara. Sin embargo, el porqué de este tipo diferencial de funcionamiento en ambos hemisferios sigue suscitando dudas. Algunos autores son partidarios de una distinta implicación de ambos hemisferios en el procesamiento de la información emocional a partir de las diferentes categorías de emociones: las formas más primitivas de emoción, que por regla general tienen connotación negativa, se encontrarían relacionadas con el funcionamiento del hemisferio derecho, mientras que aquellas otras emociones más avanzadas, y con connotaciones sociales, se encuentran especialmente vinculadas al funcionamiento del hemisferio izquierdo.

Otros autores creen que, más que en las categorías emocionales, habría que centrarse en el nivel o grado de procesamiento de la información para entender las distintas emociones, así como el papel que en ellas realiza cada uno de los hemisferios.

Uno de los autores que parece que mejor ha descrito la relevancia del hemisferio derecho en el procesamiento de la información emocional ha sido Damasio ${ }^{2}$ (Damasio, 1994, 1995, 1998; Adolphs y Damasio, 2000), quien defiende que el procesamiento de la emoción depende del 
procesamiento de la información somática. Es decir, la emoción implica unas aferencias desde el cuerpo, e implica también unas eferencias hacia el cuerpo, en ambos casos incluyendo la participación de los aspectos endocrinos y viscerales.

\subsection{Fisiología y psicología de la emoción. Consciencia de emoción}

Uno de los aspectos que más interés suscitan en al ámbito de la Psicología en general es el ámbito de la consciencia, que es el campo en el cual la emoción adquiere la connotación de experiencia emocional.

Hay autores (LeDoux, 1996; Bradley y Lang, 2000) que en los últimos tiempos proponen que la experiencia emocional perturba el verdadero conocimiento del proceso emocional, el cual se refiere a la dimensión biológica de las emociones, mediante la que se puede entender la propia evolución, a través de sus características comunes en múltiples especies de la escala filogenética. En cambio, hay otros (Clore, 1994; Damasio, 1994, 2000; Heilman, 1997, 2000) para quienes no se puede entender el conocimiento completo de un proceso emocional sin contemplar la relevancia de la dimensión subjetiva o experiencia emocional.

También los estudios de Antonio Damasio arrojan luz sobre este aspecto. En los últimos años, este neurólogo ha dedicado un gran esfuerzo en su intento por localizar las bases neurobiológicas del sentimiento emocional.

Para Damasio, la consciencia es algo enteramente privado del individuo que la posee, que ocurre como parte de un proceso, también privado y personal de ese individuo, al que denominamos mente. Pero, por otra parte, la consciencia y la mente se encuentran íntimamente asociadas a las conductas externas que manifiesta dicho individuo. Esto es, cada individuo comparte estos tres fenómenos: mente, consciencia -como parte de la mente- y conductas observables. Por otra parte, la mente y las conductas observables se encuentran también directamente asociadas con el funcionamiento de ese organismo como un todo, específicamente con el funcionamiento del cerebro de ese individuo, con lo cual nos encontramos con una tríada básica -mente, cerebro y conducta observable-, que ha permitido el avance del conocimiento en los últimos años.

En última instancia, la relación esencial se produce entre el cerebro y la mente. Ahora bien, como señala Damasio (1998), no se puede desarrollar una perspectiva integrada de la mente y el cerebro humanos si no consideramos el estudio de la emoción, y eso es posible porque la actitud actual hacia la emoción en el ámbito de la investigación ha cobrado gran interés.

En la actualidad, la emoción y su expresión representan las manifestaciones más directas de primer orden para entender la biorregulación de un organismo complejo, sobre todo cuando éste se encuentra inmerso en un ambiente con aspectos tan complejos como la cultura y la sociedad. Dicha regulación, en opinión de Damasio, no se puede entender sin apelar al papel vital que juega la emoción y posee las connotaciones de adaptación y supervivencia de los organismos que han alcanzado las más altas cotas de desarrollo, entre los que se encuentra el ser humano.

Pero la emoción también juega un papel importante en otros procesos básicos directamente relacionados con la adaptación y la supervivencia: por un lado repercute de forma clara sobre los procesos de aprendizaje, de manera que la unión entre emoción y memoria incrementa las probabilidades que tiene un organismo de adaptarse y sobrevivir, y por otro lado, también influye sobre los procesos de razonamiento y de toma de decisiones, desde las más simples hasta las más complejas. Este aspecto ha sido tratado por varios autores (Oatley, 1992; Thagard y 
Millgram, 1995; Thagard y Verbeurgt, 1998), quienes, a partir de los planteamientos de Damasio (1994), proponen una teoría emocional relacionada con la toma de decisiones.

La emoción estaría directamente relacionada con la elección de aquella alternativa de respuesta que, desde un punto de vista biológico, mejor permite al organismo conseguir la adaptación. Funcionaría como una especie de filtro que reduce apreciablemente la cantidad de información, optimizando aquellas alternativas de respuesta que, al menos aparentemente, mejor permiten al organismo adaptarse a las exigencias del medio ambiente.

De hecho, argumentan los autores, actualmente se conoce el papel fundamental que juega la corteza prefrontal en la toma de decisiones; igualmente, aunque con ciertas reticencias, se propone que la amígdala es una estructura esencial en las emociones; por lo tanto, la unión entre la corteza prefrontal ventromedial y la amígdala podría configurar un circuito neurobiológico que permitiese defender la propuesta del papel que juega la emoción en la toma de decisiones. Ese circuito podría ser considerado como la estructura neuroanatómica implicada en la conexión entre emoción y cognición.

En suma, la emoción representaría la más compleja expresión de los sistemas homeostáticos de regulación. Los resultados de la emoción se encuentran extraordinariamente vinculados a la adaptación y la supervivencia de todos los organismos que disponen de tales procesos. En cierta medida, Damasio (1998) está enfatizando la dimensión motivacional de las emociones, ya que llega a proponer que las emociones pueden ser también consideradas a lo largo de las dimensiones de aproximación-evitación o apetitiva-aversiva.

Para Damasio, las emociones son definidas como patrones de respuestas químicas y neurales, cuya función es contribuir al mantenimiento de la vida en un organismo, proporcionando conductas adaptativas. Realmente, este importante papel de las emociones se fundamenta en el hecho de que las estructuras neuroanatómicas que sirven de base a los procesos emocionales son las mismas que se encargan de controlar y regular los estados corporales básicos mediante procesos concretos, tales como la homeostasis.

Están biológicamente determinadas, siendo, por tanto, procesos automáticos. No obstante, la cultura y las experiencias e influencias que recibe un individuo a lo largo de su propio desarrollo, juegan también un papel importante. Dicha influencia se puede reflejar en el plano de los estímulos desencadenantes de una emoción, así como en el plano de la expresión emocional.

Damasio distingue entre emociones primarias, emociones secundarias o sociales, y emociones de fondo. Las emociones primarias o universales son: felicidad, tristeza, miedo, ira, sorpresa y aversión/asco. En cierta medida, Damasio está fundamentando la propuesta en el carácter universal de las expresiones faciales de la emoción. Las emociones secundarias o sociales, también denominadas por Damasio "otras conductas", son: vergüenza, celos, culpa, y orgullo. Mientras que las emociones de fondo son: bienestar, malestar, calma, tensión, energía, fatiga, anticipación, desconfianza. La peculiaridad de este último tipo de emociones consiste en la naturaleza de los inductores, que suelen ser internos, y en el foco de la respuesta, que, esencialmente, es el medio ambiente interno del organismo.

En cuanto a las estructuras neuroanatómicas implicadas en los procesos emocionales, de acuerdo con otros trabajos previos (Damasio, 1994; LeDoux, 1995, 1996), el troncoencéfalo se encuentra implicado en prácticamente todas las emociones; el hipotálamo y la corteza prefrontal ventromedial parecen las estructuras que intervienen en la emoción de tristeza, aunque no 
intervienen en otras emociones, como la ira y el miedo; por su parte, la amígdala es la estructura implicada en la emoción de miedo.

La corteza cingulada anterior también parece jugar un cierto papel en los procesos emocionales, concretamente estaría relacionada con la consciencia de la emoción. Existe una cierta coincidencia cuando se habla del sustrato neurobiológico de la experiencia consciente de la emoción, proponiendo que la corteza cingulada podría jugar un papel relevante. Esta es una estructura sumamente compleja, y con numerosas funciones, no sólo juega un papel en la experiencia consciente de la emoción, sino que, además, esta estructura se encuentra implicada, entre otras funciones importantes, en la respuesta de dolor, en la conducta maternal, en el control autonómico, en los procesos de atención, y en la selección de respuesta (Vogt y Gabriel, 1993).

La ocurrencia de un proceso emocional seguiría una sucesión de eventos, que se inician, bien con la detección de un objeto o situación mediante la percepción a través de los receptores, bien con el recuerdo de ese objeto o situación; en ambos casos, el resultado es la activación de los núcleos del troncoencéfalo, el hipotálamo y la amígdala. Luego, estas estructuras liberan hormonas de varios tipos en la corriente sanguínea, que se dirigen, por una parte, hacia diversas zonas del propio cuerpo, con lo que se modificará el perfil del medio ambiente interno, y, por otra parte, hacia distintas zonas cerebrales, tales como la corteza somatosensorial y la corteza cingulada, con lo que se modificará la señalización de los estados corporales en el cerebro.

Al mismo tiempo, estas estructuras envían, de modo simultáneo, señales electroquímicas mediante neurotransmisores, por una parte, hacia las glándulas adrenales, que liberarán hormonas con repercusión posterior en el cerebro, y, por otra parte, hacia otras regiones cerebrales, tales como la corteza, el tálamo, y los ganglios basales, con lo cual se modificará el estado cognitivo, dando lugar a la eventual manifestación de conductas emocionales, así como a una particular forma de procesar la información.

\subsection{Sentimiento y emoción. Sentimiento del sentimiento: consciencia del sentimiento.}

En la teoría de Damasio cobra especial relevancia la relación entre sentimiento y emoción, siendo necesario distinguir entre ambas variables. Son dos términos que, como hemos reseñado en varias ocasiones anteriormente, han sido utilizados de forma intercambiable por distintos autores: sin ir más lejos, el propio James (1884, 1890). También Damasio (1998, 2003: 33-41) señala que, aunque se encuentran íntimamente asociados, no son la misma cosa. Concretamente, la emoción se refiere a una serie de respuestas que, desencadenadas desde zonas concretas del cerebro, tienen lugar en otras zonas del cerebro, así como en otras partes diversas del resto del cuerpo.

El resultado final de tales respuestas es un estado emocional, que podría ser definido como el conjunto de los diferentes cambios corporales que experimenta el individuo en cuestión. Por su parte, el sentimiento se refiere al resultado del estado emocional, que, en palabras de Damasio, hace referencia a un complejo estado mental.

Este estado mental incluye, por una parte, la representación de los cambios que están ocurriendo en el propio cuerpo, y que son representados en las correspondientes estructuras del sistema nervioso central, $y$, por otra parte, diversas alteraciones en el procesamiento cognitivo, que son el resultado de las respuestas cerebro-cerebro. Es decir, primero ocurre la emoción, cuyos resultados son de dos tipos: por una parte, hacia afuera, en forma de diversas conductas, fundamentalmente en forma de expresiones más o menos definidas, que sirven para comunicar a los demás nuestro estado interno; por otra parte, hacia adentro, en forma de experiencia subjetiva 
del estado emocional o sentimiento, que afecta a la dinámica del pensamiento en curso, y, consiguientemente, a las distintas actividades cognitivas y conductas varias del futuro inmediato.

Dicho con otras palabras: el sentimiento de la emoción es la experiencia mental y privada de la emoción, mientras que la emoción es un conjunto de manifestaciones, algunas de las cuales son perfectamente observables.

Tanto la emoción como el sentimiento son susceptibles de investigación, aunque la emoción resulta bastante más asequible que el sentimiento, ya que el estímulo puede ser fácilmente identificable, pudiendo apreciar también que muchas de las manifestaciones o respuestas son externas, con lo que es mucho más viable la medida de las mismas.

El autor señala más específicamente el proceso seguido desde que un estímulo desencadena un proceso emocional hasta que un individuo toma conciencia del sentimiento producido por dicha emoción.

Así, el primer paso tiene que ver con un estado de emoción, que puede ser desencadenado y ejecutado de forma no consciente; el segundo paso tiene que ver con un estado de sentimiento, que puede ser representado no conscientemente; el tercer paso se refiere a un estado de sentimiento hecho consciente, que ocurre cuando un organismo conoce que está experimentando una emoción y un sentimiento.

Este matiz, discutible o no, es importante en la teoría de Damasio (2000), quien últimamente señala que, con el sustrato neural de la emoción, es suficiente para que ocurra un proceso emocional y el sentimiento asociado al mismo, entendiendo en este caso que el sentimiento hace referencia a una imagen mental.

El proceso seguiría el esquema siguiente:

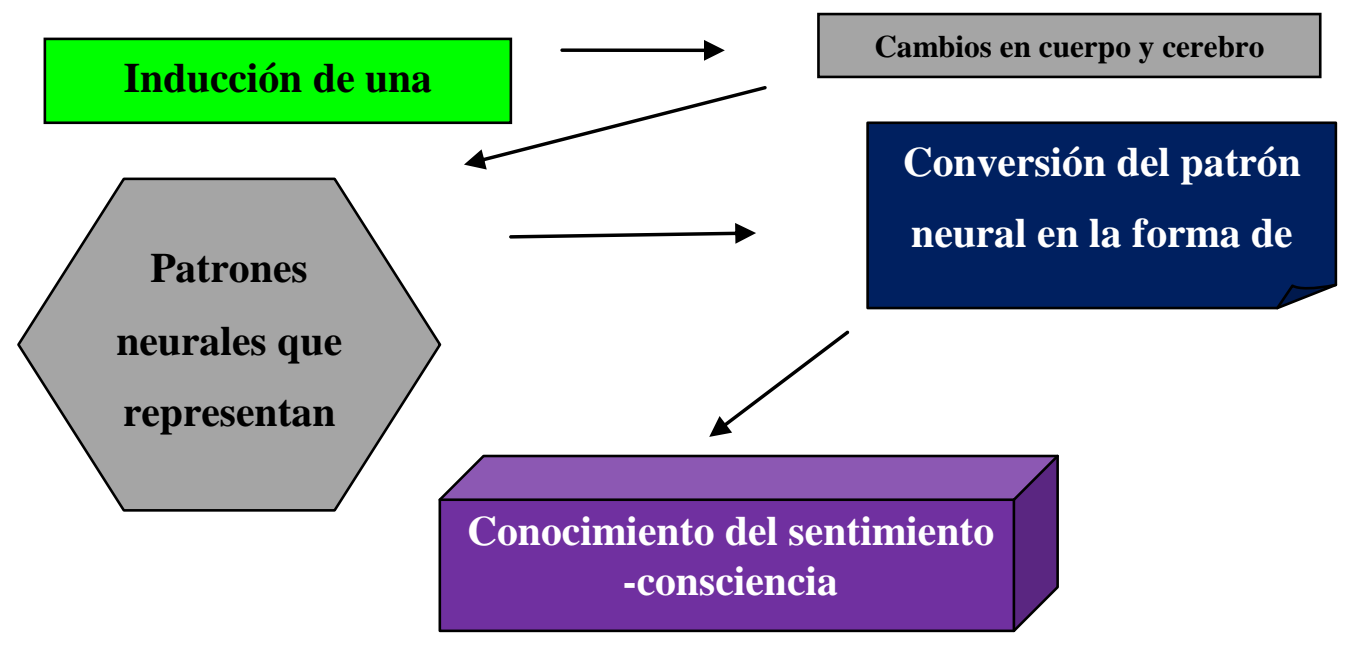

Esquema de elaboración propia 
En primer lugar, cuando se produce un estímulo, externo o interno, la corteza sensorial hace un análisis de dicho objeto o situación -o lo hace el hipocampo si se trata del recuerdo de un objeto o situación-, produciéndose al mismo tiempo la activación de las estructuras neuroanatómicas que se encuentran relacionadas con la emoción: fundamentalmente, el troncoencéfalo, el hipotálamo y la amígdala.

En segundo lugar, la activación de estas estructuras produce tres efectos: ocasiona importantes reacciones autonómicas en el cuerpo; desencadena el envío de mensajes neurales a otras zonas del cerebro; junto con la corteza somatosensorial, produce la representación de las reacciones somáticas que dichas estructuras han producido.

Finalmente, con la participación de la corteza cingulada anterior, el tálamo, se produce el mapeo del objeto junto con el siempre cambiante mapa del organismo. Este fenómeno concreto constituye lo que Damasio denomina "centro de la consciencia".

Las estructuras básicas-troncoencéfalo, hipotálamo y amígdala- parecen ser necesarias y suficientes para la ocurrencia de la emoción, pero no son suficientes para la consciencia de la emoción.

Se han hecho algunas objeciones a Damasio que tienen que ver con la ausencia de una explicación clara del modo mediante el cual un sujeto, en un estado de activación autonómica, con contracciones viscerales, incrementos en su frecuencia cardíaca, etc., es capaz de encontrar una explicación a su estado o situación, sin la información derivada de la naturaleza del estímulo. Es decir, sin saber si la información que se está procesando de ese estímulo es relevante para el bienestar del sujeto.

Esta laguna en la argumentación de Damasio es más evidente cuando el autor se refiere a las emociones secundarias o sociales. Mosca (2000) señala que no se puede entender en este planteamiento cómo es posible sentir orgullo, vergüenza o culpa sin tener en mente la representación valorativa, no sólo del objeto, sino también de las situaciones complejas que dan lugar a dichas emociones. Todo esto es debido a que Damasio no es cognitivista.

Esta filiación no cognitivista de Damasio, puede encontrar su raíz en Descartes que también negó la naturaleza representacional de los estados emocionales, considerándolos como las sensaciones que tiene la mente de los eventos cerebrales, los cuales, a su vez, son causados por la estimulación sensorial (Descartes, 1649/1985, artículo 27). Por esa razón, como indica Mosca (2000), tras aproximadamente treinta años de investigación en Psicología Cognitiva, la teoría de Damasio se encuentra bastante próxima a los clásicos argumentos de James $(1884,1890)$. Se encontraría en la línea comenzada por el empirismo inglés, y la ilustración escocesa. La diferencia entre ambos planteamientos se sitúa en el grado de conocimiento que ambos autores (James y Damasio) poseen acerca de la relación entre procesos emocionales y cerebro, siendo el de Damasio mucho mayor que el de James.

A nuestro juicio, el análisis de Damasio es neurológicamente muy completo, pero quizá falle al dar un paso más y llegar a la consciencia. La deuda con el empirismo es estar demasiado pegados a la materialidad de las funciones cognoscitivas. Otro camino es recoger todos los elementos que ha descubierto la neurología actual sin despreciarlos y acudir a otras fuentes para dar el paso a la consciencia. 


\section{Interactividad, comunicación y emociones.}

\subsection{La esencia de la comunicación digital: la interactividad}

Ante cualquier tipo de información, puede haber una respuesta emocional, pero para que haya comunicación no basta con que se sienta una emoción, sino que hay que procesarla, y lograr que llegue a ser consciente. Las respuestas emocionales dependen de múltiples factores, entre ellos de los medios de transmisión de información a los que se recurra. Entre los medios, cabe destacar algunos factores que pueden influir en las respuestas emocionales, como la velocidad con la que se transmite la información, la exigencia de una respuesta inmediata, o la interpelación al espectador.

Para ver mejor cómo se desenvuelven las emociones en las redes sociales, analizaremos brevemente cuáles son las características principales de dichas redes, y de su rasgo esencial que es la interactividad.

En primer lugar resumiremos cómo se ha realizado la evolución de la web en la red, para poder dilucidar en qué momento las emociones realizan su aparición de una manera diferente a lo que habitualmente estamos acostumbrados a percibir en la vida cotidiana.

La web 1.0, llamada también web estática, utiliza las tecnologías HTML, XML, etc. Hizo posible una web que podríamos llamar de lectura, en la que los contenidos son introducidos por personas concretas, y el resto del público accede a esos contenidos a modo informativo. Está estructurada sobre documentos y enlaces de hipertexto, y fue diseñada para la lectura humana y no para que su información se pudiera procesar de forma automática.

La web 2.0, o web social, permite que el usuario genere sus propios contenidos y comparta sus producción; como consecuencia, surgen herramientas de comunicación, con nuevos servicios basados en la colaboración en línea, y se forman comunidades para compartir información. Como ejemplo se pueden citar los Weblog o bitácoras, los podcast, los sistemas de RSS, las wikis y otras aplicaciones orientadas a proveer un servicio social. Ejemplos de sitios y empresas que utilizan la web 2.0 son Wikipedia, Flickr, MySpace o YouTube.

Ya se está hablando de la web 3.0 pero es un concepto todavía sin determinar. Parece ser que se basará en una Internet más "inteligente", los usuarios podrán hacer búsquedas más cercanas al lenguaje natural, la información tendrá semántica asociada y la web podrá relacionar conceptos de múltiples fuentes, también podrá deducir información a través de reglas asociadas al significado del contenido. Por este motivo la web 3.0 supondrá otra era de búsqueda de información

Esta denominación de web 3.0 genera cierta resistencia, pues todavía no se ha generalizado el uso de la web 2.0 en todos los niveles sociales. Para algunos, es la evolución natural de la web 2.0; para otros, no guarda ninguna relación, pues es una nueva web. Quizá no se entienda lo mismo al hablar de web 3.0 desde diversas instancias. Hay quienes la entienden como sinónimo de la web3D, una extensión de los mundos virtuales que hoy ya existen en Internet, como Second Life. Otro ejemplo sería Google Earth, que nos permite, con solo un clic, visitar distintas ciudades del globo. La web 3D sería aquella donde podemos recrear el mundo donde existimos.

Otros la entienden como una web que está en todas partes: ordenadores, móviles, o incluso en algunas pantallas o espejos situados en las casas. Por último, estarían aquellos que la 
entenderían como una web semántica, como Tim Berners-Lee, el creador de Internet, intuyó en sus inicios y lo dio a conocer públicamente en la revista Scientific American en 2001.

Esta web semántica hace referencia a la capacidad de los servidores web para comprender el contenido de todo lo que almacenan o distribuyen por la red, ya sea bajo la forma de texto, sonido, imagen o gráficos. Se habla de una web "inteligente" capaz de entender el lenguaje y buscar datos sin intervención directa del ser humano; una web donde las máquinas puedan discernir y jerarquizar el conocimiento que existe en la red. $\mathrm{Y}$ esto para algunos supone un cambio fundamental porque no se trata solo de buscar sino de lograr objetivos. Así, Mari Carmen Marcos preguntaba a Ricardo Baeza-Yates: “¿Cree que la web semántica, tal como la planteó Tim Berners-Lee, será realidad algún día? En ese caso ¿qué papel deberán jugar los buscadores en la web semántica?". La respuesta fue la siguiente (Marcos, 2008):

"Espero que sea realidad algún día. Sin embargo el problema actual es más social que tecnológico, pues implica que las personas deben ser consistentes en las metodologías y herramientas de creación de páginas web, lo que es difícil de asegurar. También todavía no existen los estándares que permitan determinar si un sitio web tiene información semántica válida o no. Por supuesto que si esto ocurre buscar será mucho más fácil, ya que no habrá que adivinar la semántica, obteniendo resultados mucho mejores"

Para desarrollar esta web harán falta otros lenguajes como son el RDF Resource Description Framework, el OWL o web Ontology Language, que puede escribir clases, propiedades y estados de algo, y el XML o Extensible Markup Language, que facilita el entendimiento de los datos entre distintos sistemas informáticos. Estos lenguajes son la base sintáctica de la web Semántica.

Según las concepciones de la web 3.0 se verá a ésta como una simple herramienta para poner orden en Internet y buscar con más facilidad las cosas, o como una nueva restructuración de la web desde sus cimientos. Incluso algunos aventuran la posibilidad de una red al modo de cerebro humano que pueda aprender y pensar a modo de cerebro global. Todavía es algo que está en sus comienzos.

En resumen, podríamos decir que la web 1.0 se relaciona con las emociones del individuo de manera similar a como lo haría un libro o un periódico; solo ha cambiado el medio de transmisión, pero el tipo de lenguaje es el mismo. Incluso en ocasiones se podría asemejar al cine cuando se introduce sonido (Segura García y Martínez Rodrigo, 2010). El individuo está recibiendo de modo pasivo un mensaje que puede provocar una respuesta emocional.

Sin embargo, en el caso de la web 2.0, nos encontramos ante una comunicación diferente. El individuo participa de modo activo, y en ocasiones se exige una respuesta; el sujeto elabora su propia producción. En el caso de la web 3.0, la dinámica red-usuario estaría por ver, pero parece que iría en la misma línea que las web 2.0; es decir estaríamos ante algo muy parecido a una conversación, pero con unos matices -que no por eso dejan de ser cruciales en nuestro estudiodiferentes.

La interactividad también es una aspiración de los actuales canales de televisión, que de hecho están desarrollando mejoras en esa dirección:

"Abrir el abanico de posibilidades de poder interactuar con la televisión ha sido una vieja aspiración que ha llevado a la puesta en práctica de fórmulas tecnológicas limitadas y poco eficaces, sobre todo desde que la Red ha venido a proporcionarnos unas posibilidades de 
elección ilimitadas. Así como la televisión le ha disputado el terreno al cine, el ordenador, a través de la Red es una fuente inagotable de entretenimiento e información que hace de menos a la televisión. Por eso es previsible que se produzca una integración de ambas plataformas. La televisión ganaría en interactividad, el ordenador se beneficiaría de la espectacularidad de los nuevos formatos de imagen para televisión." (Hernández, 2011)

Como se observa, los medios de comunicación han alcanzado un gran desarrollo tecnológico. Pero esta transformación, ¿supone meramente un cambio instrumental o se está hablando de una mutación más profunda que afecta a la propia comunicación?

Resulta muy lúcido el análisis que realiza Scolari (2008) acerca de la digitalización de la comunicación. Este autor hace un recorrido entre los estudios de comunicación de masas y las nuevas formas de comunicación mediadas por tecnologías digitales, en el que nos vamos a apoyar para entender mejor la relación actual entre comunicación e interactividad y de qué tipo de transformación estamos hablando.

Scolari percibe que la llegada de la revolución digital ha desencadenado una crisis entre los modelos teóricos tradicionales de la comunicación. Para resolver tal crisis, plantea una oposición entre las formas tradicionales de comunicación masiva y las nuevas formas de comunicación digital. Dicha oposición se plantea desde una arqueología semántica acerca de términos como digitalizaciones, hipertextualidades, reticularidades, interactividades, mutimedialidades, convergencias y remedaciones,

Dichos términos constituirán los rasgos de la nueva forma de comunicación. Y así, el concepto de hipermediaciones queda definido por Scolari como "procesos de intercambio, producción y consumo simbólico que se desarrollan en un entorno caracterizado por una gran cantidad de sujetos, medios y lenguajes interconectados tecnológicamente de manera reticular entre sí" (2008: 113-114).

Lo que está proponiendo es un salto semántico al reflexionar sobre el concepto de hipermediación, y sugiere: "perder la fascinación por los nuevos medios, para recuperar las hipermediaciones" (2008: 116); reivindica de esta forma el rol activo protagonista del receptor tradicional, transformado ahora en figura híbrida como 'prosumidor', como este autor lo denomina, es decir, simultáneamente productor y consumidor (2008: 98). Considera necesario generar nuevos paradigmas teóricos que permitan entender lo que está pasando en el mundo de las comunicaciones digitales interactivas.

Resulta por tanto necesario reelaborar los conceptos tradicionales de emisor, receptor, audiencia y usuario, es decir, las nuevas figuras de la comunicación digital, para explicar bien qué es, por ejemplo, el ciberusuario y a qué construcciones sociales hace referencia.

Para Scolari, todas estas transformaciones no son meramente tecnológicas sino que afectan al mundo y a la comprensión de éste por parte del sujeto. En ese sentido, para el autor, una "teoría de las hipermediaciones, debe saber moverse en un terreno discursivamente pantanoso, consolidando una sólida red de interlocutores a partir de los cuales comenzar a construir su propio recorrido epistemológico" (2008: 144).

Según este autor, nos encontramos ante una mutación muy significativa de la sociedad del siglo XXI: la aparición del ámbito comunicativo digital interactivo. 
Y ¿cómo se podría definir la interactividad? Roberto Igarza (2008) realiza un buen análisis de este concepto definiéndola como la capacidad de crear una situación de intercambio entre los usuarios. Otorga al usuario la posibilidad de intervenir y originar mediante esa intervención una modificación en el contexto o en la consecución del diálogo o de la navegación. Gracias a la interactividad, los usuarios pueden interactuar para elegir su propia trayectoria y combinar recursos a su disposición con el fin de obtener la información deseada; la interactividad incide de modo definitivo en la comunicación.

Todo esto desde el punto de vista del usuario, pero desde el punto de vista del sistema, la interactividad lleva consigo la disposición de todo tipo de facilidades para que el usuario pueda influir en el comportamiento del sistema en línea de la manera más individualizada posible.

¿Cuáles son los rasgos de la interactividad? Podrían resumirse en los siguientes: inmediatez, personalización, ampliación, no linealidad y participación (Muñoz, 2010). Pasamos a describirlos brevemente.

La "inmediatez" introduce un cambio de escala y ritmo, tendiendo a la instantaneidad. El usuario puede acceder a la información cuando quiere y en lo que quiere, sin tener que atenerse a un horario como ocurre, por ejemplo, en la programación de la televisión analógica. Lo característico de este tipo de comunicación es una enorme cercanía entre los usuarios y los hechos sobre los que se han informado, lo cual lleva a una complejidad creciente. En este rasgo late una necesidad cada vez mayor de calidad de las informaciones, puesto que la rapidez no es sinónimo de calidad. Respecto a la "personalización", cabe destacar que es el usuario quien selecciona cómo, dónde y cuándo desea recibir la información, a la vez que escoge las informaciones de mayor relevancia para él. De alguna manera, el usuario puede convertirse en realizador en la medida en que utiliza servicios que le permiten configurar un menú de contenidos, o elige la cámara desde donde observar un evento.

La "ampliación" se refiere a que el usuario puede complementar sus contenidos según sus deseos; no se trata de una competencia de contenidos, sino que se va ampliando el conocimiento.

La "no linealidad", por su parte, viene a indicar que el orden de navegación no es único, que no hay solo una ruta para acceder a contenidos, sino que puede haber varias posibilidades de enlaces.

Por último, el rasgo llamado "participación" destaca que el usuario es de alguna manera productor de su contenido. El estatuto del usuario ya no es pasivo, sino activo, que llega a su culmen cuando se establece una red y la participación ya no es de un usuario aislado, sino de varios usuarios activos entre sí.

\section{2. Dinámica de las emociones en redes sociales}

Hemos visto las características generales de lo que se podría llamar una comunicación digital y de la interactividad, rasgo esencial de las redes sociales. También hemos analizado un recorrido neurológico y psicológico de las emociones. Nos encontramos pues, en disposición de estudiar a continuación cómo se desenvolverían las emociones en redes sociales teniendo en cuenta las características de estas últimas.

El ser humano es multidimensional: en él se integran aspectos sensitivos, afectivos, intelectivos o racionales, sin estar desligados unos de otros. $Y$ a todas estas dimensiones debemos remitirnos a 
la hora de analizar la comunicación humana para llegar a conclusiones no sesgadas, que reflejen lo que en realidad acontece en el acto de comunicación. Resultan muy interesantes las observaciones de la Dra. López Moratalla (2011) acerca de la relación entre el mundo de las relaciones éticas y su correlato fisiológico en el cerebro, que aportan elementos de interés para nuestra investigación: la ética no es tanto un producto del cerebro como al revés. El cerebro está preparado para sistematizar conductas éticas: es decir, hay una correlación entre anatomíafisiología y capacidades que posee el hombre a la hora de relacionarse con el entorno.

Acudiendo de nuevo al neurólogo portugués, Antonio Damasio (2009), en una de sus observaciones en este terreno publicada en la revista Proceedings of the National Academy of Science, señala a nivel neurológico cómo se desenvuelven las emociones con algunas herramientas de Internet: imágenes de violencia y sufrimiento en televisión, medios digitales cada vez más veloces y difíciles de procesar, relaciones personales sustituidas por redes sociales como Facebook o Twitter... ¿Cómo podría influir todo esto en las capacidades cognitivas del ser humano? ¿Procesamos de igual manera las emociones cuando nos llegan a través de un formato digital?

Las conclusiones de este investigador proceden de un experimento llevado a cabo con 13 voluntarios de la Universidad de Southern California (en Los Ángeles, EEUU), donde Damasio dirige el Instituto del Cerebro y la Creatividad.

Después de escuchar historias reales que trataban de despertar en ellos sentimientos tanto de admiración y de compasión en el sentido físico como de empatía social, se observó lo que ocurría en su cerebro mediante imágenes de resonancia magnética. Hasta ahora los estudios cerebrales sobre la compasión estaban limitados a los sentimientos que despierta en nosotros el dolor ajeno; ésta es la primera vez que se extiende este concepto en un sentido más amplio de compasión o empatía social y se aborda además la admiración. Es decir, estamos ante la misma realidad que definía Adam Smith como ponerse en el lugar de otro.

Los sentimientos relacionados con cuestiones morales y psicológicas -admiración por un buen acto o una habilidad- tardaron más en activarse en el cerebro que los relacionados con cuestiones físicas: ante un accidente, por ejemplo.

Sin embargo, si los sentimientos "sociales" tardaron más en surgir -de seis a ocho segundos- que las reacciones ante estímulos físicos como el daño ajeno -unas décimas de segundo-, los investigadores también descubrieron que duraban más tiempo de modo activo en el cerebro de los participantes. Esto pone de manifiesto que el cerebro es capaz de distinguir perfectamente las emociones que tienen que ver con las cuestiones físicas de aquellas que suscitan las dimensiones morales o psicológicas de una situación.

Trasladando el problema a las redes sociales por ejemplo, nos encontramos con que las emociones que surgen de la empatía social tardan en aparecer pero duran más; en cambio, si el instrumento es Twitter, por ejemplo, o cualquier otra red social, dada la rapidez de la comunicación, surge la duda de si el cerebro humano está preparado para producir emociones adecuadas a esa velocidad. La clave está en que la empatía social necesita cierta ponderación o reflexión. Con la velocidad de las redes sociales, apenas da tiempo a que se elabore dicha reflexión. 
Acudiendo a los análisis de Scolari y Muñoz, podemos entender mejor por qué es más compleja la comunicación esencialmente interactiva, digital, que la comunicación tradicional, como sería una conversación.

De las características propias de la interactividad, parece que tres son las que más relación pueden tener con el desencadenamiento de las emociones:

La inmediatez, que se halla relacionada con la rapidez o velocidad en recibir información y dar una respuesta, que a veces comporta una gran complejidad;

- La personalización: el usuario se halla en un contexto determinado, situación que influye directamente en la selección del momento, lugar y modo de acceso a la información;

- $\quad$ La participación: se invita continuamente a interactuar, a responder y a producir contenido.

Revisando dichos rasgos, podemos decir que estos son catalizadores que potencian la respuesta emocional del usuario en las redes sociales de por sí interactivas. Quizá el rasgo más importante que marca la diferencia es la complejidad que surge en la inmediatez y la velocidad: una conversación verbal puede ser rápida, pero siempre es bidireccional, mientras que una comunicación interactiva en redes puede ser multidireccional y con múltiples vínculos de acceso a una nueva información.

Esto nos lleva a concluir que las emociones no pueden funcionar de la misma manera en una relación basada en los instrumentos de las redes sociales, por ejemplo, que en una relación interpersonal presencial o "real", por llamarla de alguna manera. Este aspecto resulta particularmente importante en los jóvenes y niños que ya han comenzado a realizar sus primeros pasos en la comunicación y en la educación con medios digitales, puesto que comienzan a configurar el universo y a desarrollar sus capacidades tanto humanas, como cognitivas o emocionales, desde dichos medios (Sábada, 2005).

\section{Conclusiones}

Las conclusiones a las que podemos llegar tras este estudio pormenorizado acerca de qué son y cómo funcionan las emociones y cómo se desenvuelve la comunicación en las redes sociales, son muy variadas:

1) Las emociones forman parte de la comunicación humana, con independencia de las herramientas que se utilicen en el proceso comunicativo.

2) La dinámica por la que el cerebro procesa una emoción es diferente si se trata de una emoción provocada por algo físico, o si es fruto de la reacción ante un hecho moral. La diferencia está en la tardanza en aparecer y en su duración. Son más inmediatas y duran menos las emociones ante hechos físicos, que las emociones ante realidades morales o psicológicas.

3) Además, sentimiento y emoción tienen un reflejo diferente en el cerebro. Aquel es más duradero, se refiere más bien a un estado ánimo, y es más complejo de procesar, especialmente en el caso de llegar a una consciencia del mismo. La emoción es más inmediata e inconsciente.

4) El uso de herramientas propias de la web 2.0 aporta un aumento de velocidad en la gestión de la información. Esto puede hacer que se pueda intercambiar información y establecer una relación sin que dé tiempo a que aparezca un sentimiento consciente. 
5) Si esto es así, no tener en cuenta la velocidad en la comunicación de información puede hacer desaparecer una dimensión propia del ser humano. Los rasgos de la interactividad de una comunicación digital no son los mismos que los de una interacción propia de una conversación hablada: la complejidad de ésta última es mucho menor.

6) Esto lleva a pensar que, además de saber utilizar tecnológicamente las herramientas digitales, hay que aprender a usar las capacidades cognitivas humanas, de manera que las herramientas se adapten a nuestras capacidades y no al revés, con el fin de que haya una buena comunicación.

7) La causa de esta, llamémosla así, desnaturalización, no estaría propiamente en la aparición de las herramientas digitales sino el uso que se haga de ellas.

8) Esto nos lleva a considerar que la enseñanza de TIC no debe ser meramente técnica, sino que debe incluir otro tipo de aprendizaje. Es decir, el individuo debe saber cómo gestionar la velocidad de la información. Es posible que haya personas que, ante esa falta de "tiempo", no puedan elaborar la respuesta adecuada. Por ejemplo, la respuesta ante un herido en un accidente es inmediata, apenas necesita procesar la respuesta. Sin embargo, la respuesta ante un comentario en Facebook, puede no ser tan inmediata, pues falta el contexto, el tono de voz, la situación en la que se ha escrito aquello, etc. Es frecuente contestar, tomar decisiones en red (compras por ejemplo, participación en encuestas con premio), o forjarse una opinión acerca de un hecho sin contrastar, llevados por la inercia de la velocidad sin tener en cuenta este factor.

9) La emotividad es un elemento que enriquece el conocimiento; a veces supone un mecanismo de defensa frente a lo perjudicial, como se ha visto en el origen filogenético de las emociones, y en otras ocasiones, un estímulo frente a lo conveniente. Perder este rasgo puede ponernos en situación de vulnerabilidad -no tanto física, como psicológica- sin darnos cuenta.

10) El aprendizaje de las TIC, tanto en niveles de educación inferiores como superiores, debería tener en cuenta estos factores psicológicos relacionados con la interactividad, de manera que el aprendizaje no sea meramente técnico sino humano, y que las TIC sean realmente herramientas del individuo y no al revés, que el individuo se configure en torno a una herramienta.

\section{Bibliografía}

BERICAT, E. (2004): "La sociología de la emoción y la emoción en la sociología". Papers, nº 62, pp. $145-176$

BRADLEY, M. y LANG, P. (2000): "Measuring emotion: Behavior, feeling, and physiology" en R.D. Lane y L. Nadel (eds.): Cognitive Neuroscience of Emotion, Nueva York: Oxford University Press. pp. 242-276.

CLORE, G. (1994): "Why emotions are felt" en P. Ekman y R.J. Davidson (eds.): The Nature of Emotion: Fundamental Questions, Nueva York: Oxford University Press. pp. 103-111.

DAMASIO, A. (2009): "Cerebral and spinal modulation of pain by emotions". Proceedings of the National Academy of Science, vol. 106, № 49.

DAMASIO, A. (2003): En busca de Spinoza, neurobiología de la emoción y de los sentimientos. Barcelona: Ed. Crítica. 


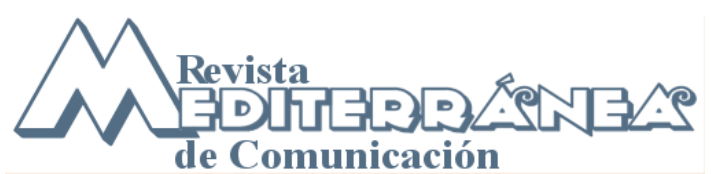

ISSN 1989-872X - Año2 (2011), pp. 171- 190

DAMASIO, A. (1994): Descartes' error: Emotions, Reason, and the Human Brain. Nueva York: Avon Books.

DAMASIO, A. (1995): "Toward a neurobiology of emotion and feeling: Operational concepts and hypotheses". The Neuroscientist, no 1, pp.19-25.

DAMASIO, A. (1998): "Emotion in the perspective of an integrated nervous system". Brain Research Reviews, no 26, pp. 83-86.

DAMASIO, A. (2000): "A second chance for emotion" en R.D. Lane y L. Nadel (eds.): Cognitive Neuroscience of Emotion. Nueva York: Oxford University Press, pp. 12-23.

DESCARTES, R. (trad. 1985): Pasiones del Alma. Meditaciones metafísicas. Madrid: Orbis.

DIXON, T. (2003): From Passions to emotions. Cambridge: Cambridge University Press.

ESTEBANEL, M. (2002): “Interactividad e interacción”, en RELATEC: Revista Latinoamericana de Tecnología Educativa, ISSN 1695-288X, Vol. 1, №. 1, págs. 15-25.

GOLDIE, P. (2000): Las emociones: una exploración filosófica. Oxford: Clarendon Press.

HEILMAN, K. (1997): "The neurobiology of emotional experience". Journal of Neuropsychiatry and Clinical Neurosciences, no 9, pp. 439-448.

HEILMAN, K. (2000): "Emotional experience: A neurological model" en R.D. Lane y L. Nadel (eds.): Cognitive Neuroscience of Emotion. 2000. Nueva York: Oxford University Press. pp. 328344.

HERNÁNDEZ RODRÍGUEZ, C.E. (2011): "Historia de la competencia entre el cine y la televisión. Los efectos de las nuevas tecnologías en la formas de consumir y producir cine en España: la explosión del cine en la televisión y el posible fin de las películas en celuloide" en Revista Mediterránea de Comunicación, año 2, [consultado: 7 de julio de 2011]

HOCHSCHILD, A. (2008): La mercantilización de la vida íntima. Apuntes de la casa y el trabajo. Barcelona: Katz.

IGARZA, R. (2008): Nuevos medios, estrategias de convergencia. Buenos Aires: La Crujía.

ILLOUZ, E. (2009): El consumo de la utopía romántica. Barcelona: Katz.

JAMES, W. (1890): Principles of Psychology. Nueva York: Holt.

KENNY, A. (1969): Action, emotion and will. London: Routledge \& Kegan Paul.

LEDOUX, J. (1995): "Emotion: Clues from the brain”. Annual Review of Psychology, n 46, 209235.

LEDOUX, J. (1996): The Emotional Brain: the Mysterious Underpinnings of Emotional Life. Nueva York: Simon and Schuster. 
LÓPEZ MORATALLA, N. (2011): "El cerebro ético, como atajo emocional ante dilemas", en La crónica de la ciencia, marzo: www.blogs.lainformacion.com/cronicas-de-la-ciencia/2011/03/10/elcerebro-etico-como-atajo-emocional-ante-dilemas/ [Consultado: 20/05/2011]

MACH, E. (1987): Análisis de las sensaciones. Barcelona: Ed. Alta Fulla.

MARCOS, M.C. (2008): "Entrevista a Ricardo Baeza-Yates de Yahoo! Investigación" [on line]. Hipertext.net, núm. 6. http://www.hipertext.net/web/pag290.htm [Consulta: 19/05/2010]

MARTÍNEZ RODRIGO, E. (2010): "Lectores dinámicos ante textos interactivos". Revista Icono14 [en línea] 15 de Enero de 2010, № 15. pp. 261-273 http://www.icono14.net 2010. [Consultado: 12 de septiembre de 2010]

MOSCA, A (2004): "A Review Essay on Antonio Damasio's "The Feeling of What Happens: Body and Emotion in the Making of Consciousness". Psyche, 6(10). http://www.theassc.org/files/assc/2468.pdf [Consultado: 28/05/2011]

MUÑOZ, G. (2010): “¿De los nuevos medios a las hipermediaciones?”. Revista latinoamericana de ciencias sociales, vol. 8 (1), p. $9-16, \quad$ separata, http://www.umanizales.edu.co/publicaciones/campos/cinde/Vol8Separata/pdf\%20completo\%20Se parata.pdf [consultado 13 de julio de 2011]

NUSSBAUM, M. (1997): Justicia poética. Barcelona: Andrés Bello.

OATLEY, K. (1992): Best Laid Schemes: The Psychology of Emotions. Cambridge: Cambridge University Press.

SÁBADA, Ch. y BRINGUÉ, X. (2005): Nacidos digitales, una generación frente a las pantallas. Madrid: Rialp.

SCOLARI, C. (2008): Hipermediaciones. Elementos para una Teoría de la Comunicación Digital Interactiva. Barcelona: Editorial Gedisa, S.A.

SEGURA GARCÍA, R. y MARTÍNEZ RODRIGO, E. (2010): “Música y sentimiento en los medios audiovisuales". Razón y Palabra, http://www.razonypalabra.org.mx/N/N73/Varia73/22SeguraMartinez_V73.pdf [Consultado: 10/08/2010].

SMITH, A. (trad. 1974): The theory of moral sentiments. Indianapolis: Liberty Foundation.

THAGARD, P. y MILLGRAM, E. (1995): "Inference to the best Plan: A Coherence Theory of Decision", en A. Ram y D. B. Leake (eds.): Goal-Driven Learning. Cambridge, MA: MIT Press. pp. 439-454.

THAGARD, P. y VERBEURGT, K. (1998): "Coherence as constraint satisfaction". Cognitive Science, no 22, pp. 1-24.

TELLIS, G. y REDONDO, I (2000): Estrategias de publicidad y promoción. Madrid: Pearson Education. 
VOGT, B.A y GABRIEL M. (eds.) (1993): Neurobiology of Cingulate Cortex and Limbic Thalamus. Boston: Birkhauser.

WOLTON, D. (2005): Pensar la comunicación. Buenos Aires: Prometeo.

YEPES, R. y ARANGUREN, J. (2004): Fundamentos de antropología humana. Pamplona: EUNSA.

\section{Notas}

\footnotetext{
${ }^{1}$ Es el caso de Thomas Sheff, Arlie R. Hochschild, Theodor D. Kemper; en España, Eduardo Bericat ha realizado un análisis de esta situación. .cfr. HOSCHILD (2008), BERICAT (2000)

2 Damasio. es profesor de la cátedra David Dornsife de Psicología, Neurociencia y Neurología en la Universidad del Sur de California, donde dirige el Institute for the Neurological Study of Emotion and Creativity de los Estados Unidos Es un conocido investigador en varias áreas de las neurociencias. Sus intereses se centran en las bases neurológicas de la mente, sobre todo en lo que se refiere a los sistemas neuronales relacionados con la memoria, el lenguaje, las emociones y las decisiones. Uno de los libros más recientes de Damasio, Looking for Spinoza: joy, sorrow, and the feeling brain fue publicado en 2003. En él, Damasio analiza la relación de la filosofía con la neurobiología así como la relación entre la ética humana y la ciencia.
} 